\title{
Review of: "Self-assembled Nanoparticles of Polycationic $b$ Rush and $p$ Hotosensitizer for Synergistic Photothermal and Photodynamic Therapies Against Periodontitis"
}

\author{
Xin $\mathrm{Li}^{1}$ \\ 1 Rheinisch Westfälische Technische Hochschule Aachen
}

Potential competing interests: The author(s) declared that no potential competing interests exist.

\section{Comments}

This work investigated the synthesis of self-assembled nanosystem based on polycationic brushes (sPDMA) and ICG for synergistic PTT and PDT against periodontitis. The research is extremely well and a lot of experimental data are offered to support the conclusion. However, some important issues should be addressed.

1. Please provide peak integration in the NMR plot (Fig. 1a and S2).

2. Page 6, Line 18-19, the detailed mechanism of nanoprecipitation method for the self-assembly of ICG and SPDMA shoud be described (through electrostatic or hydrophobic interaction?).

3. The authors claim that the nanosystem exhibits the synergistic effect of PTT and PDT against periodontitis. Therefore, the synergistic index should be calculated in the manuscript, and the related calculation method can refer to the literature (Journal of Advanced Research, 2021,

DOI:10.1016/i.jare.2021.08.004) ${ }^{[1]}$

4. Page 4 in Supplementary Information, "The photothermal performances... ... at the ICG and SPDMA concentrations of $35 \mathrm{ug} / \mathrm{mL}$ and $1.25 \mathrm{mg} / \mathrm{mL}$, respectively." is mentioned. However, the ICG/sPDMA weight ratio is $4 / 14$. The authors should explain the choice of the concentrations of ICG ( $35 \mathrm{ug} / \mathrm{mL}$ ) and sPDMA (1.25 mg/mL).

5. In Fig. 2a, the unit should be ug/mL instead of um. Moreover, for the temperature decrease after $4 \mathrm{~min}$, the authors should give some explanation. Finally, the temperature changes of pure ICG solution upon laser irradiation should be provided.

6. The experimental method regarding the result in Fig. $3 \mathrm{c}$ is missing in Supplementary Information.

7. Page 9, Line 6-9, “After 808 nm laser irradiation..., free ICG and sPDMA@ICG NPs both inhibited the bacterial growth remarkably, ... (Fig. 4d)." is mentioned. However, from Fig. 4d, we can clearly see that when ICG concentration is lower than $5 \mathrm{ug} / \mathrm{mL}$, the ICG $+\mathrm{L}$ group does not have significant antibacterial effect. Therefore, the authors should revise the relevant description.

8. In Fig. $6 \mathrm{~d}$, the infrared thermal images of periodontal tissues before laser irradiation should be provided. 
9. The author's choice of laser irradiation duration ( $5 \mathrm{~min}$ or $10 \mathrm{~min}$ ) caused me serious confusion. In the in vivo experiment, the irradiation duration is $5 \mathrm{~min}$. While the laser irradiation duration is $10 \mathrm{~min}$ in the in vitro experiment (e.g., Fig. 4a-c, e-f). Notably, in Fig. 4d, the irradiation duration returns to 5 min. The authors should unify the laser irradiation time or give some explanations.

10. In the in vivo experiment (i.e., Fig.6-7), the SPDMA and SPDMA+L groups were added as control. Similarly, these two control groups should also be added to the in vitro experiment (i.e., Fig. 4-5). 11. Page 10, Line 19-22, “As shown in Fig. 6d, sPDMA@ICG NPs increased the temperature of periodontal issue rapidly to $43.1^{\circ} \mathrm{C}$ within 1 min of laser irradiation and $51.2^{\circ} \mathrm{C}$ within 5 min of laser irradiation... this temperature will not bring about the serious damages to the normal tissues" is mentioned. According to the previous reports (Techniques in Vascular and Interventional Radiology, 2013, 16(4), 192-200. Advanced Functional Materials, 2021, 31(26), 2011227.), ${ }^{[2][3]}$ the temperature of about $50^{\circ} \mathrm{C}$ will cause the irreversible damage and significant necrosis for cell in vivo. Likewise, we also do not find the relevant description in ref.38. ${ }^{[4]}$ Therefore, the authors should be careful to declare their views.

12. The laser intensity used in the research work $\left(808 \mathrm{~nm}, 2 \mathrm{~W} / \mathrm{cm}^{2}\right)$ is significantly higher than the maximum permissible exposure value of skin $\left(808 \mathrm{~nm}, 0.33 \mathrm{~W} / \mathrm{cm}^{2}\right)$ according to the standard reported by the American National Standards Institute. ${ }^{[5]}$ The authors may consider using safer laser power to complete the treatment in future research.

13. The references mentioned above should be cited in the manuscript.

\section{References}

1. `Xin Li, Lingdan Kong, Wei Hu, Changchang Zhang, et al. (2021). Safe and efficient 2D molybdenum disulfide platform for cooperative imaging-guided photothermal-selective chemotherapy: A preclinical study. Journal of Advanced Research. doi:10.1016/j.jare.2021.08.004.

2. `Erica M. Knavel, Christopher L. Brace. (2013). Tumor Ablation: Common Modalities and General Practices. Techniques in Vascular and Interventional Radiology, vol. 16 (4), 192-200. doi:10.1053/j.tvir.2013.08.002.

3. `Xin Li, Haitao Sun, Helin Li, Chaolei Hu, et al. (2021). Multi-Responsive Biodegradable Cationic Nanogels for Highly Efficient Treatment of Tumors. Adv. Funct. Mater.. doi:10.1002/adfm.202100227.

4. 'Yuanyuan Cheng, Qian Chen, Zhaoyang Guo, Mengwen Li, et al. (2020). An Intelligent Biomimetic Nanoplatform for Holistic Treatment of Metastatic Triple-Negative Breast Cancer via Photothermal Ablation and Immune Remodeling. ACS Nano, vol. 14 (11), 15161-15181. doi:10.1021/acsnano.0c05392.

5. American National Institute. , , , , et al. (2007). American National Standard for the Safe Use of Lasers ANSI Z136.1.. HE Standard for Laser Safety. Laser Institute of America: NewYork. 